\title{
CLOSEDNESS OF COBOUNDARY MODULES OF ANALYTIC SHEAVES
}

\author{
BY \\ YUM-TONG SIU AND GÜNTHER TRAUTMANN( ${ }^{(1)}$
}

\begin{abstract}
Suppose $A$ is a subvariety of a complex space $X$ and $\mathscr{F}$ is a coherent analytic sheaf on $X$. It is shown that, if the analytic sheaf $\mathscr{H}_{A}^{\nu}(\mathscr{F})$ of local cohomology is coherent for $0 \leqq \nu<q$, then for $0 \leqq \nu \leqq q$ the local cohomology group $H_{A}^{v}(X, \mathscr{F})$ with its natural topology is Hausdorff and hence is a Fréchet space.
\end{abstract}

Suppose $\mathscr{F}$ is a coherent analytic sheaf on a (not necessarily reduced) complex space $X$. Denote by $S_{k}(\mathscr{F})$ the subvariety $\left\{x \in X \mid \operatorname{codh} \mathscr{F}_{x} \leqq k\right\}$ [4, Satz 5]. For any open subset $D$ of $X$, denote by $\bar{S}_{k}(\mathscr{F} \mid D)$ the topological closure of $S_{k}(\mathscr{F} \mid D)$ in $X$. If $A$ is a subvariety of $X$, denote by $\mathscr{H}_{A}^{k}(\mathscr{F})$ the sheaf defined by the presheaf $U \mapsto H_{A}^{k}(U, \mathscr{F})$, where $H_{A}^{k}(U, \mathscr{F})$ is the $k$-dimensional cohomology group of $U$ with coefficients in $\mathscr{F}$ and supports in $A$. If $\phi: X \rightarrow Y$ is a holomorphic map of complex spaces, denote by $\phi_{k}(\mathscr{F})$ the $k$ th direct image of $\mathscr{F}$ under $\phi$.

In [6] and [9] the following coherence criterion for sheaves of local cohomology is proved:

THEOREM 1. Suppose $A$ is a subvariety of a complex space $X, q$ is a nonnegative integer, and $\mathscr{F}$ is a coherent analytic sheaf on $X$. Let $\theta: X-A \rightarrow X$ be the inclusion map. Then the following three statements are equivalent:

(i) $\theta_{v}(\mathscr{F} \mid X-A)$ is coherent on $X$ for $0 \leqq \nu \leqq q$.

(ii) $\mathscr{H}_{A}^{v}(\mathscr{F})$ is coherent on $X$ for $0 \leqq \nu \leqq q+1$.

(iii) $\operatorname{dim} A \cap \bar{S}_{k+q+1}(\mathscr{F} \mid X-A)<k$ for $k \geqq 0$.

A trivial modification of the proof of [1, Satz 5] yields readily the following isomorphism theorem:

TheOREM 2. Suppose $Y$ and $Z$ are complex spaces and $Z$ is Stein. Suppose $\pi: Y \rightarrow Z$ is a holomorphic map and $\mathscr{G}$ is a coherent analytic sheaf on $Y$. If $q$ is a nonnegative integer and $\pi_{\nu}(\mathscr{G})$ is coherent on $Z$ for $0 \leqq \nu<q$, then for $0 \leqq \nu \leqq q$ the natural homomorphism $H^{\nu}(Y, \mathscr{G}) \rightarrow \Gamma\left(Z, \pi_{\nu}(\mathscr{G})\right)$ is an isomorphism.

Received by the editors November 24, 1969.

AMS 1969 subject classifications. Primary 3250; Secondary 3227.

Key words and phrases. Coherent analytic sheaves, local cohomology, coboundary modules, Fréchet spaces.

(1) During the research of this paper the first author was partially supported by NSF Grant GP-7265, and the second author was a Fulbright Scholar.

Copyright (C) 1970, American Mathematical Society 
For a coherent analytic sheaf $\mathscr{F}$ on a complex space $X$ (with countable topology), we give $H^{v}(X, \mathscr{F})$ the quotient topology induced by $Z^{v}(\mathfrak{u}, \mathscr{F})$, where $\mathfrak{u}$ is a (countable) Stein open covering of $X$. This quotient topology on $H^{v}(X, \mathscr{F})$ can be easily verified to be independent of the choice of $\mathfrak{u}$. Suppose $X$ is Stein and $A$ is a subvariety of $X$. For $\nu>1$ we give $H_{A}^{v}(X, \mathscr{F})$ the topology induced through the algebraic isomorphism $H_{A}^{v}(X, \mathscr{F}) \approx H^{v-1}(X-A, \mathscr{F}) . \quad H_{A}^{1}(X, \mathscr{F})$ is given the quotient topology induced by $\Gamma(X-A, \mathscr{F})$ through the algebraic isomorphism

$$
H_{A}^{1}(X, \mathscr{F}) \approx \Gamma(X-A, \mathscr{F}) / \operatorname{Im}(\Gamma(X, \mathscr{F}) \rightarrow \Gamma(X-A, \mathscr{F})) .
$$

$H_{A}^{\circ}(X, \mathscr{F})$ is given the topology induced by $\Gamma(X, \mathscr{F})$ through the algebraic isomorphism

$$
H_{A}^{0}(X, \mathscr{F}) \approx \operatorname{Ker}(\Gamma(X, \mathscr{F}) \rightarrow \Gamma(X-A, \mathscr{F}))
$$

Denote by $N_{A}^{v}(X, \mathscr{F})$ the topological closure of 0 in $H_{A}^{\nu}(X, \mathscr{F})$.

In view of Theorems 1 and 2 , it is natural to raise the following questions: If one of the three equivalent statements in Theorem 1 is satisfied and $X$ is Stein, is the algebraic isomorphism

$$
H^{v}(X-A, \mathscr{F}) \rightarrow \Gamma\left(X, \theta_{v}(\mathscr{F} \mid X-A)\right)
$$

also a topological isomorphism for $0 \leqq \nu \leqq q$ (where $\Gamma\left(X, \theta_{v}(\mathscr{F} \mid X-A)\right.$ ) as the section module of a coherent analytic sheaf is given the natural Fréchet structure)? Is $H^{q+1}(X-A, \mathscr{F})$ a Fréchet space? Some special cases of these questions are considered in [9]. In this paper we give an affirmative answer to the above questions.

MAIN TheOREM. Suppose $\mathscr{F}$ is a coherent analytic sheaf on a complex space $X$ and $A$ is a subvariety of $X$. Suppose $\mathscr{H}_{A}^{v}(X, \mathscr{F})$ is coherent on $X$ for $0 \leqq \nu \leqq q$. Then

(i) $H_{A}^{v}(X, \mathscr{F})$ is a Fréchet space for $0 \leqq \nu \leqq q+1$,

(ii) the algebraic isomorphism $H_{A}^{v}(X, \mathscr{F}) \rightarrow \Gamma\left(X, \mathscr{H}_{A}^{v}(\mathscr{F})\right)$ is a topological isomorphism for $0 \leqq \nu \leqq q$, and

(iii) $\mathscr{H}_{A}^{q+1}(\mathscr{F})$ is a Fréchet sheaf [2, VIII. A.3].

The proof of the Main Theorem is obtained by refining the proof of Theorem 1 and keeping track of all the topologies involved there.

In this paper complex spaces are not necessarily reduced and all of them have countable topology. All linear topological spaces are over $\boldsymbol{C}$ and need not be Hausdorff. ${ }_{n} \mathcal{O}$ denotes the structure sheaf of $C^{n}$.

Suppose $A$ is a subvariety of a Stein complex space $X$. Let $\mathfrak{U}$ be a Stein open covering of $X-A$. For a coherent analytic sheaf $\mathscr{F}$ on $X$, define the $(-1)$ th cochain group $C^{-1}(\mathfrak{U}, \mathscr{F})$ as $\Gamma(X, \mathscr{F})$ and define the coboundary map $\delta: C^{-1}(\mathfrak{U}, \mathscr{F})$ $\rightarrow C^{0}(\mathfrak{u}, \mathscr{F})$ by restrictions. Define $C^{-2}(\mathfrak{u}, \mathscr{F})=0$ and define $\delta: C^{-2}(\mathfrak{u}, \mathscr{F})$ 
$\rightarrow C^{-1}(\mathfrak{u}, \mathscr{F})$ as the zero map. Using these notations, we have the following natural topological isomorphism:

$$
H_{A}^{q}(X, \mathscr{F}) \approx Z^{q-1}(\mathfrak{u}, \mathscr{F}) / B^{q-1}(\mathfrak{U}, \mathscr{F})
$$

Lemma 1. Suppose $A$ is a subvariety of a Stein complex space $X$ and $q$ is a nonnegative integer.

(a) If $\mathscr{F}$ is a coherent analytic sheaf on $X$ and $X^{\prime}$ is a Stein open subset of $X$, the linear map $\rho: H_{A}^{q}(X, \mathscr{F}) \rightarrow H_{A^{\prime}}^{q}\left(X^{\prime}, \mathscr{F}\right)$ induced by restriction is continuous, where $A^{\prime}=A \cap X^{\prime}$.

(b) If $\lambda: \mathscr{F} \rightarrow \mathscr{G}$ is a sheaf-homomorphism of coherent analytic sheaves on $X$, then the linear map $\lambda^{*}: H_{A}^{q}(X, \mathscr{F}) \rightarrow H_{A}^{q}(X, \mathscr{G})$ induced by $\lambda$ is continuous.

(c) If $0 \rightarrow \mathscr{F}^{\prime} \rightarrow \mathscr{F} \rightarrow \mathscr{F}^{\prime \prime} \rightarrow 0$ is an exact sequence of coherent analytic sheaves on $X$, then the linear map $\delta^{*}: H_{A}^{q}\left(X, \mathscr{F}^{\prime \prime}\right) \rightarrow H_{A}^{q+1}\left(X, \mathscr{F}^{\prime}\right)$ in the cohomology sequence of the exact sequence is continuous. Moreover, if $H$ is a closed subspace of $H_{A}^{q+1}\left(X, F^{\prime}\right)$ contained in $\operatorname{Im} \delta^{*}$, then the map $\left(\delta^{*}\right)^{-1}(H) \rightarrow H$ induced by $\delta^{*}$ is open.

Proof. Let $\mathfrak{u}$ be a Stein open covering of $X-A$.

(a) Let $\mathfrak{u}^{\prime}$ be a Stein open covering of $X^{\prime}-A^{\prime}$ which refines $\mathfrak{u}$. The continuity of $\rho$ follows from the continuity of the restriction map $Z^{q-1}(\mathfrak{U}, \mathscr{F}) \rightarrow Z^{q-1}\left(\mathfrak{U}^{\prime}, \mathscr{F}\right)$.

(b) The continuity of $\lambda^{*}$ follows from the continuity of the map $Z^{q-1}(\mathfrak{u}, \mathscr{F})$ $\rightarrow Z^{q-1}(\mathfrak{u}, \mathscr{F})$ induced by $\lambda$.

(c) The exact sequence $0 \rightarrow \mathscr{F}^{\prime} \rightarrow \mathscr{F} \rightarrow \mathscr{F}^{\prime \prime} \rightarrow 0$ gives rise to the following commutative diagram with exact rows:

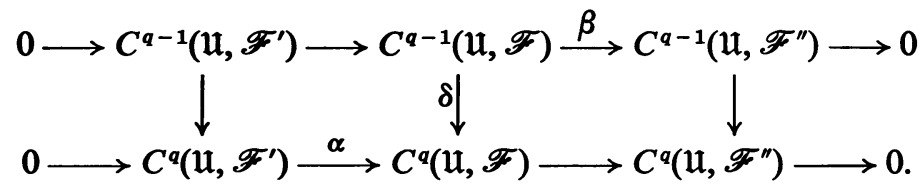

Let $\sigma: Z^{q-1}\left(\mathfrak{u}, \mathscr{F}^{\prime \prime}\right) \rightarrow H_{A}^{q}\left(X, \mathscr{F}^{\prime \prime}\right)$ be the natural map. Consider the following commutative diagram:

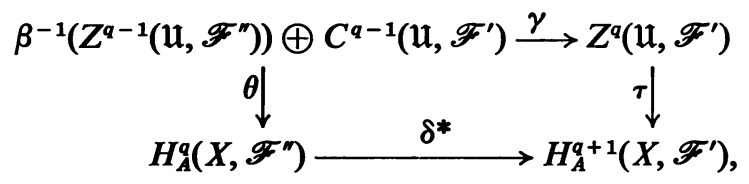

where $\theta(a \oplus b)=\sigma(\beta(a)), \gamma(a \oplus b)=\alpha^{-1}(\delta(a))+\delta(b)$, and $\tau$ is the natural map.

$\theta$ and $\tau$ are continuous and open. The continuity $\delta^{*}$ follows from the continuity of $\gamma$. Since $\tau^{-1}\left(\operatorname{Im} \delta^{*}\right)=\operatorname{Im} \gamma$, if $H$ is a closed subspace of $H_{A}^{q+1}\left(X, \mathscr{F}^{\prime}\right)$ contained in $\operatorname{Im} \delta^{*}$, then the openness of the map $\left(\delta^{*}\right)^{-1}(H) \rightarrow H$ induced by $\delta^{*}$ follows from applying the open mapping theorem for Fréchet spaces to the map $\gamma^{-1}\left(\tau^{-1}(H)\right)$ $\rightarrow \tau^{-1}(H)$ induced by $\gamma$. Q.E.D. 
LEMMA 2. Suppose A is a subvariety of a Stein complex space $X$ and $\mathscr{F}$ is a coherent analytic sheaf on $X$ such that $\mathscr{H}_{A}^{0} \mathscr{F}=0$. Suppose $X^{\prime}$ is a Stein open subset of $X$ such that $X-X^{\prime}$ is a subvariety of $X$. Let $A^{\prime}=A \cap X^{\prime}$ and $A^{\prime \prime}=A-X^{\prime}$. Then there exists an exact sequence

$$
\begin{aligned}
0 \longrightarrow H_{A^{\prime \prime}}^{1}(X, \mathscr{F}) \longrightarrow H_{A}^{1}(X, \mathscr{F}) \stackrel{\alpha_{1}}{\longrightarrow} H_{A^{\prime}}^{1}\left(X^{\prime}, \mathscr{F}\right) \longrightarrow H_{A^{\prime \prime}}^{2}(X, \mathscr{F}) \longrightarrow \cdots \\
\longrightarrow H_{A^{\prime \prime}}^{v}(X, \mathscr{F}) \longrightarrow H_{A}^{v}(X, \mathscr{F}) \stackrel{\alpha_{v}}{\longrightarrow} H_{A^{\prime}}^{v}\left(X^{\prime}, \mathscr{F}\right) \longrightarrow H_{A^{\prime \prime}}^{v+1}(X, \mathscr{F}) \longrightarrow \cdots,
\end{aligned}
$$

where $\alpha_{\nu}$ is induced by restrictions and is continuous.

Proof. The sequence is induced from the Mayer-Vietoris sequence of $\mathscr{F}$ on $X-A^{\prime \prime}=(X-A) \cup X^{\prime}$. The continuity of $\alpha_{v}$ follows from Lemma 1 (a). Q.E.D.

LEMMA 3. If $X$ is a Stein open subset of $C^{n}$ and $A$ is a subvariety of dimension $r$ in $X$, then $H_{A}^{n-r}\left(X,{ }_{n} \mathcal{O}\right)$ is Hausdorff.

Proof. We can assume that $r<n$.

(a) Consider first the special case where

and

$$
X=\left\{\left(z_{1}, \ldots, z_{n}\right) \in C^{n}|| z_{i} \mid<1,1 \leqq i \leqq n\right\}
$$

$$
A=\left\{\left(z_{1}, \ldots, z_{n}\right) \in X \mid z_{i}=0, r+1 \leqq i \leqq n\right\} .
$$

Let $H=\left\{\left(z_{1}, \ldots, z_{n}\right) \in X \mid z_{i} \neq 0, r+1 \leqq i \leqq n\right\}$. Let $B$ be the subset of all elements $f$ of $\Gamma\left(H,{ }_{n} \mathcal{O}\right)$ whose Laurent series expansions in $z_{r+1}, \ldots, z_{n}$ have the form

$$
f=\sum\left\{f_{v_{r+1} \cdots v_{n}} z_{r+1}^{v_{r+1}} \cdots z_{n}^{v_{n}} \mid \nu_{i} \geqq 0 \text { for some } r+1 \leqq i \leqq n\right\} .
$$

$B$ is a closed subspace of $\Gamma\left(H,{ }_{n} \mathcal{O}\right)$. It is easily verified that $H_{A}^{n-r}\left(X,{ }_{n} \mathcal{O}\right)$ is topologically isomorphic to $\Gamma\left(H,{ }_{n} \mathcal{O}\right) / B$ and hence is Hausdorff.

(b) Assume that $A$ is nonsingular and has pure dimension $r$. Since $\mathscr{H}_{A}^{v}\left({ }_{n} \mathcal{O}\right)=0$ for $0 \leqq \nu<n-r$ [3, Korollar zu Satz 3], by Theorem 2, the canonical homomorphism $H_{A}^{n-r}\left(X,{ }_{n} \mathcal{O}\right) \rightarrow \Gamma\left(X, \mathscr{H}_{A}^{n-r}\left({ }_{n} \mathcal{O}\right)\right)$ is an isomorphism. Hence we need only prove that every point of $A$ admits an open neighborhood $U$ in $X$ such that $H_{A \cap U}^{n-r}\left(U,{ }_{n} \mathcal{O}\right)$ is Hausdorff, but this follows from (a).

(c) For the general case, choose a holomorphic function on $X$ whose zero-set $B$ satisfies the following two conditions:

(i) $\operatorname{dim} B \cap A<r$ and

(ii) $A-B$ is nonsingular and has pure dimension $r$.

Let $X^{\prime}=X-B, A^{\prime}=A-B$, and $A^{\prime \prime}=A \cap B$. By Lemma 2, we have the following exact sequence:

$$
0=H_{A^{n}}^{n-r}\left(X,{ }_{n} \mathcal{O}\right) \longrightarrow H_{A}^{n-r}\left(X,{ }_{n} \mathcal{O}\right) \stackrel{\alpha}{\longrightarrow} H_{A^{\prime}}^{n-r}\left(X^{\prime},{ }_{n} \mathcal{O}\right),
$$

where $\alpha$ is continuous. Since by (b) $H_{A^{n}}^{n-r}\left(X^{\prime},{ }_{n} \mathcal{O}\right)$ is Hausdorff, $H_{A}^{n-r}\left(X,{ }_{n} \mathcal{O}\right)$ is Hausdorff. Q.E.D.

REMARK. Lemma 3 is a special case of $[7,(4.1)]$. 
Proposition 1. Suppose $X$ is a Stein open subset of $C^{n}, A$ is a subvariety of dimension $r$ in $X, \mathscr{F}$ is a coherent analytic sheaf on $X$, and $0 \leqq q \leqq n-r$. Let $\mathscr{I}$ be the ideal-sheaf of $A \cup S_{r+q-1}(\mathscr{F})$. If $X^{\prime}$ is a relatively compact Stein open subset of $X$, then there exists a nonnegative integer $k=k\left(X^{\prime}\right)$ such that $\Gamma\left(X^{\prime}, \mathscr{I}\right)^{k} N_{A^{q}}^{q}\left(X^{\prime}, \mathscr{F}\right)=0$ and $\Gamma\left(X^{\prime}, \mathscr{O}\right)^{k} H_{A^{\prime}}^{v}\left(X^{\prime}, \mathscr{F}\right)=0$ for $0 \leqq \nu<q$, where $A^{\prime}=A \cap X^{\prime}$.

Proof. (a) First we reduce the general case to the special case $q=n-r$. Suppose $0 \leqq q<n-r$. By shrinking $X$, we can assume w.l.o.g. that we have the following exact sequence of coherent analytic sheaves on $X$ :

$$
0 \rightarrow \mathscr{G} \rightarrow{ }_{n} \mathcal{O}^{p_{n-r-q-1}} \rightarrow{ }_{n} \mathcal{O}^{p_{n-r-q-2}} \rightarrow \cdots \rightarrow{ }_{n} \mathcal{O}^{p_{1}} \rightarrow{ }_{n} \mathcal{O}^{p_{0}} \rightarrow \mathscr{F} \rightarrow 0 .
$$

Since $H_{A^{\prime}}^{\nu}\left(X^{\prime},{ }_{n} \mathcal{O}\right)=0$ for $0 \leqq \nu \leqq n-r-1$ [3, Korollar zu Satz 3], the exact sequence yields algebraic isomorphisms

$$
H_{A^{\prime}}^{v}\left(X^{\prime}, \mathscr{F}\right) \stackrel{\approx}{\longrightarrow} H_{A^{\prime}}^{v+n-r-q}\left(X^{\prime}, \mathscr{G}\right) \text { for } 0 \leqq \nu<q
$$

and an algebraic monomorphism

$$
\lambda: H_{A^{\prime}}^{q}\left(X^{\prime}, \mathscr{F}\right) \rightarrow H_{A^{\prime}}^{n-r}\left(X^{\prime}, \mathscr{G}\right) .
$$

By Lemma $1, \lambda$ is continuous. $\lambda\left(N_{A^{\prime}}^{q}\left(X^{\prime}, \mathscr{F}\right)\right) \subset N_{A^{\prime}}^{n-r}\left(X^{\prime}, \mathscr{G}\right)$. Since $S_{n-1}(\mathscr{G})$ $=S_{r+q-1}(\mathscr{F})$, the general case is reduced to the special case $q=n-r$.

(b) Suppose $q=n-r$. By applying the functor $\operatorname{Hom}_{n} \mathcal{O}\left(\cdot,{ }_{n} \mathcal{O}\right)$ to a free resolution of $\operatorname{Hom}_{n} \mathcal{O}\left(\mathscr{F},{ }_{n} \mathcal{O}\right)$ of length $n-r+1$ and combining it with the natural sheafhomomorphism $\mathscr{F} \rightarrow \operatorname{Hom}_{n} \mathcal{O}\left(\operatorname{Hom}_{n} \mathcal{O}\left(\mathscr{F},{ }_{n} \mathcal{O}\right),{ }_{n} \mathcal{O}\right)$, after shrinking $X$, we can assume w.l.o.g. that we have the following order-two sequence of coherent analytic sheaves on $X$ which is exact on $X-S_{n-1}(\mathscr{F})$ :

$$
\begin{aligned}
& 0 \longrightarrow \mathscr{F} \stackrel{\varphi_{-1}}{\longrightarrow}{ }_{n} \mathcal{O}^{s_{0}} \stackrel{\varphi_{0}}{\longrightarrow}{ }_{n} \mathcal{O}^{s_{1}} \stackrel{\varphi_{1}}{\longrightarrow} \cdots \\
& \stackrel{\varphi_{n-r-1}}{\longrightarrow}{ }_{n} \mathcal{O}^{s_{n-r}} \stackrel{\varphi_{n-r}}{\longrightarrow}{ }_{n} \mathcal{O}^{s_{n-r+1}} .
\end{aligned}
$$

By Hilbert Nullstellensatz, there exists a nonnegative integer $l$ such that

$$
\mathscr{I}^{l} \mathscr{H}_{A}^{0}\left(\operatorname{Ker} \varphi_{\nu}\right)=0 \text { on } X^{\prime} \text { for }-1 \leqq \nu \leqq n-r
$$

and

$$
\mathscr{I}^{\prime}\left(\operatorname{Ker} \varphi_{v} / \operatorname{Im} \varphi_{\nu-1}\right)=0 \text { on } X^{\prime} \text { for }-1 \leqq \nu \leqq n-r,
$$

where $\operatorname{Im} \varphi_{-2}=0$.

We are going to prove (3) $)_{v}$ and $(4)_{v}$ for $0 \leqq \nu \leqq n-r$ by induction on $\nu$ :

(3) $)_{v} \Gamma\left(X^{\prime}, \mathscr{A}\right)^{(\mu+1) l} H_{A^{\prime}}^{\mu}\left(X^{\prime}, \operatorname{Ker} \varphi_{n-r-v}\right)=0$ for $0 \leqq \mu \leqq \min (v, n-r-1)$.

(4)v $\Gamma\left(X^{\prime}, \mathscr{I}^{(\mu+2) l} H_{A^{\prime}}^{\mu}\left(X^{\prime}, \operatorname{Im} \varphi_{n-r-v-1}\right)=0\right.$ for $0 \leqq \mu \leqq \min (\nu, n-r-1)$. 
Because of (1), (3) $)_{0}$ is true. (3) implies (4) $)_{v}$, because of the exactness of

$$
\begin{aligned}
H_{A^{\prime}}^{\mu-1}\left(X^{\prime}, \operatorname{Ker} \varphi_{n-r-v} / \operatorname{Im} \varphi_{n-r-v-1}\right) \rightarrow H_{A^{\prime}}^{\mu}\left(X^{\prime}, \operatorname{Im} \varphi_{n-r-v-1}\right) & \\
& \rightarrow H_{A^{\prime}}^{\mu}\left(X^{\prime}, \operatorname{Ker} \varphi_{n-r-v}\right)
\end{aligned}
$$

which comes from the exact sequence

$$
0 \rightarrow \operatorname{Im} \varphi_{n-r-v-1} \rightarrow \operatorname{Ker} \varphi_{n-r-v} \rightarrow \operatorname{Ker} \varphi_{n-r-v} / \operatorname{Im} \varphi_{n-r-v-1} \rightarrow 0
$$

and because of (2). (4) implies (3) $)_{v+1}$, because of (1) and because of the exactness of

$$
H_{A^{\prime}}^{\mu}\left(X^{\prime}, \operatorname{Im} \varphi_{n-r-v-1}\right) \rightarrow H_{A^{\prime}}^{\mu+1}\left(X^{\prime}, \operatorname{Ker} \varphi_{n-r-v-1}\right) \rightarrow H_{A^{\prime}}^{\mu+1}\left(X^{\prime},{ }_{n} \mathcal{O}^{s_{n-r-v-1}}\right)=0
$$

which comes from the exact sequence

$$
0 \rightarrow \operatorname{Ker} \varphi_{n-r-v-1} \rightarrow{ }_{n} \mathcal{O}_{n-r-v-1}^{s_{n}} \rightarrow \operatorname{Im} \varphi_{n-r-v-1} \rightarrow 0 .
$$

The induction process is complete.

$(4)_{n-r}$ implies that $\Gamma\left(X^{\prime}, \mathscr{I}\right)^{(v+2) l} H_{A^{\prime}}^{\nu}\left(X^{\prime}, \mathscr{F}\right)=0$ for $0 \leqq \nu<n-r$.

Consider the following exact sequence which comes from $(6)_{n-r-1}$ :

$$
H_{A^{\prime}}^{n-r-1}\left(X^{\prime}, \operatorname{Im} \varphi_{0}\right) \stackrel{\alpha}{\longrightarrow} H_{A^{\prime}}^{n-r}\left(X^{\prime}, \operatorname{Ker} \varphi_{0}\right) \stackrel{\beta}{\longrightarrow} H_{A^{\prime}}^{n-r}\left(X^{\prime},{ }_{n} \mathcal{O}^{s_{0}}\right) .
$$

Since $\beta$ is continuous (Lemma 1$)$ and $H_{A^{\prime}}^{n-r}\left(X^{\prime},{ }_{n} \mathcal{O}^{8} 0\right)$ is Hausdorff (Lemma 3), $N_{A^{\prime}}^{n-r}\left(X^{\prime}, \operatorname{Ker} \varphi_{0}\right) \subset \operatorname{Im} \alpha$. By $(4)_{n-r-1}$, we have

$$
\Gamma\left(X^{\prime}, \mathscr{I}\right)^{(n-r+1) l} N_{A^{\prime}}^{n-r}\left(X^{\prime}, \operatorname{Ker} \varphi_{0}\right)=0 .
$$

Consider the following exact sequence which comes from $(5)_{n-r}$ :

$$
H_{A^{\prime}}^{n-r-1}\left(X^{\prime}, \operatorname{Ker} \varphi_{0} / \operatorname{Im} \varphi_{-1}\right) \stackrel{\xi}{\longrightarrow} H_{A^{\prime}}^{n-r}\left(X^{\prime}, \operatorname{Im} \varphi_{-1}\right) \stackrel{\eta}{\longrightarrow} H_{A^{\prime}}^{n-r}\left(X^{\prime}, \operatorname{Ker} \varphi_{0}\right) .
$$

Since $\eta$ is continuous,

$$
\eta\left(N_{A^{\prime}}^{n-r}\left(X^{\prime}, \operatorname{Im} \varphi_{-1}\right)\right) \subset N_{A^{\prime}}^{n-r}\left(X^{\prime}, \operatorname{Ker} \varphi_{0}\right) .
$$

By (7), we have

$$
\Gamma\left(X^{\prime}, \mathscr{I}\right)^{(n-r+1) l} N_{A^{\prime}}^{n-r}\left(X^{\prime}, \operatorname{Im} \varphi_{-1}\right) \subset \operatorname{Im} \xi .
$$

As a consequence of (2), we obtain $\Gamma\left(X^{\prime}, \mathscr{T}\right)^{(n-r+2) l} N_{A^{\prime}}^{n-r}\left(X^{\prime}, \mathscr{F}\right)=0$. Q.E.D.

Proposition 2. Suppose $X$ is a Stein open subset of $C^{n}, A$ is a subvariety in $X$, $\mathscr{F}$ is a coherent analytic sheaf on $X$, and $q$ is a nonnegative integer. Suppose $V$ is $a$ subvariety of $X$ containing $A$ such that for $k \geqq 0$ every branch of $S_{k+q}(\mathscr{F})$ not contained in $V$ intersects $A$ in a subvariety of dimension $\leqq k$. Let $I$ be the ideal-sheaf of $V$. If $X^{\prime}$ is a relatively compact Stein open subset of $X$, then there exists a nonnegative integer $l=l\left(X^{\prime}\right)$ such that $\Gamma\left(X^{\prime}, \mathscr{I}\right)^{l} N_{A^{\prime}}^{q}\left(X^{\prime}, \mathscr{F}\right)=0$, where $A^{\prime}=A \cap X^{\prime}$. 
Proof. (a) First we reduce the general case to the special case $\mathscr{H}_{V}^{0} \mathscr{F}=0$. Let $\mathscr{G}=\mathscr{F} \mid \mathscr{H}_{V}^{0} \mathscr{F}$. There exists a nonnegative integer $l$ such that $\mathscr{I}^{l} \mathscr{H}_{V}^{0} \mathscr{F}=0$ on $X^{\prime}$. The exact sequence

$$
\mathbf{0} \rightarrow \mathscr{H}_{V}^{0} \mathscr{F} \rightarrow \mathscr{F} \rightarrow \mathscr{G} \rightarrow 0
$$

yields the exact sequence

$$
H_{A^{\prime}}^{q}\left(X^{\prime}, \mathscr{H}_{\mathbf{V}}^{0} \mathscr{F}\right) \stackrel{\alpha}{\longrightarrow} H_{A^{\prime}}^{q}\left(X^{\prime}, \mathscr{F}\right) \stackrel{\beta}{\longrightarrow} H_{A^{\prime}}^{q}\left(X^{\prime}, \mathscr{G}\right) .
$$

Since $\beta$ is continuous, $\beta\left(N_{A^{\prime}}^{q}\left(X^{\prime}, \mathscr{F}\right)\right) \subset N_{A^{\prime}}^{q}\left(X^{\prime}, \mathscr{G}\right)$. If $\Gamma\left(X^{\prime}, \mathscr{I}\right)^{l^{\prime}} N_{A^{\prime}}^{q}\left(X^{\prime}, \mathscr{G}\right)=0$, then $\Gamma\left(X^{\prime}, \mathscr{I}\right)^{l^{\prime}} N_{A^{\prime}}^{q}\left(X^{\prime}, \mathscr{F}\right) \subset \operatorname{Im} \alpha$ and therefore $\Gamma\left(X^{\prime}, \mathscr{T}\right)^{l+l^{\prime}} N_{A^{\prime}}^{q}\left(X^{\prime}, \mathscr{F}\right)=0$.

(b) Suppose $\mathscr{H}_{V}^{0} \mathscr{F}=0$. We can assume $q>0$. Let $Y=\operatorname{Supp} \mathscr{F}$. We can assume $\boldsymbol{Y} \neq \varnothing$. Let $\boldsymbol{m}=\sup \left\{\operatorname{codh} \mathscr{F}_{\boldsymbol{x}} \mid x \in \boldsymbol{Y}\right\}$. Since $\mathscr{H}_{V}^{0} \mathscr{F}=0$, every branch of $\boldsymbol{Y}$ intersects $X-V$. Let $r=m-q$. Since $Y=S_{m}(\mathscr{F})$ and no branch of $Y$ is contained in $V$, $\operatorname{dim} A \cap Y \leqq r$. By replacing $A$ by $A \cap Y$, we can assume that $\operatorname{dim} A \leqq r$ and $r \geqq 0$.

For $-1 \leqq k \leqq r$ define $A_{k}$ to be the intersection of $A$ and the union of all branches of $S_{k+q}(\mathscr{F})$ not contained in $V . \operatorname{dim} A_{k} \leqq k$ and $A_{r}=A$.

Construct subvarieties $A_{k}^{*}$ of $X(-1 \leqq k \leqq r)$ by descending induction on $k$ such that

(i) $A_{r}^{*}=A_{r}$,

(ii) $A_{k} \subset A_{k}^{*}$ and $\operatorname{dim} A_{k}^{*} \leqq k$,

(iii) $A_{k}^{*}$ is the intersection of $A_{k+1}^{*}$ and the zero-set $B_{k}$ of some holomorphic function on $X$, and

(iv) $S_{k+q}(\mathscr{F}) \subset V \cup B_{k}$.

Let $A_{k}^{\prime}=X^{\prime} \cap A_{k}^{*}$ and $A_{k}^{\prime \prime}=A_{k}^{\prime}-B_{k-1}$. By Proposition 1 there exists a nonnegative integer $l$ such that

$$
\Gamma\left(X^{\prime}-B_{k}, \mathscr{T}^{l} H_{A_{k+1}}^{q-1}\left(X^{\prime}-B_{k}, \mathscr{F}\right)=0\right.
$$

and

$$
\Gamma\left(X^{\prime}-B_{k}, \mathscr{T}\right)^{l} N_{A_{\tilde{k}+1}}^{q}\left(X^{\prime}-B_{k}, \mathscr{F}\right)=0 .
$$

We are going to prove $(10)_{k}$ for $-1 \leqq k \leqq r$ by induction on $k$ :

$$
\Gamma\left(X^{\prime}, \mathscr{T}\right)^{(2 k+1) l} N_{A_{k}}^{q}\left(X^{\prime}, \mathscr{F}\right)=0 .
$$

Trivially we have (10) $)_{-1}$. Suppose $-1 \leqq k<r$ and $(10)_{k}$ is true. By Lemma 2 , we have the following exact sequence

$$
E \longrightarrow H_{A_{k}}^{q}\left(X^{\prime}, \mathscr{F}\right) \stackrel{\alpha}{\longrightarrow} H_{A_{k}+1}^{q}\left(X^{\prime}, \mathscr{F}\right) \stackrel{\beta}{\longrightarrow} H_{A_{\tilde{k}+1}}^{q}\left(X^{\prime}-B_{k}, \mathscr{F}\right)
$$

where

$$
\begin{aligned}
E & =0 \text { when } q=1 \\
& =H_{A_{k+1}}^{q-1}\left(X^{\prime}-B_{k}, \mathscr{F}\right) \quad \text { when } q>1 .
\end{aligned}
$$


Since $\beta$ is continuous,

$$
\beta\left(N_{A_{k+1}}^{q}\left(X^{\prime}, \mathscr{F}\right)\right) \subset N_{A_{\tilde{k}+1}}^{q}\left(X^{\prime}-B_{k}, \mathscr{F}\right) .
$$

By (9), we have

$$
\Gamma\left(X^{\prime}, \mathscr{P}\right)^{l} N_{A_{k+1}}^{q}\left(X^{\prime}, \mathscr{F}\right) \subset \operatorname{Im} \alpha .
$$

Choose a Stein open covering $\mathfrak{u}$ of $X^{\prime}-A_{k}^{\prime}$ and a Stein open covering $\mathfrak{B}$ of $X^{\prime}-A_{k+1}^{\prime}$ such that $\mathfrak{B}$ refines $\mathfrak{u}$. Let

$$
\eta: Z^{q-1}(\mathfrak{U}, \mathscr{F}) \rightarrow Z^{q-1}(\mathfrak{B}, \mathscr{F})
$$

be the restriction map. Define

$$
\theta: Z^{q-1}(\mathfrak{u}, \mathscr{F}) \oplus C^{q-2}(\mathfrak{B}, \mathscr{F}) \rightarrow Z^{q-1}(\mathfrak{B}, \mathscr{F})
$$

by $\theta(a \oplus b)=\eta(a)+\delta(b)$.

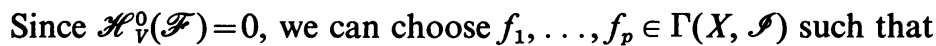

(i) $f_{1}, \ldots, f_{p}$ generate $\mathscr{I}$ on $X^{\prime}$, and

(ii) the sheaf-homomorphism $\mathscr{F} \rightarrow \mathscr{F}$ defined by multiplication by $f_{i}$ is injective.

Take $f=f_{i_{1}} \ldots f_{i_{i}}$. To finish the proof of $(10)_{k+1}$, we need only show that

$$
f \Gamma\left(X^{\prime}, \mathscr{I}\right)^{(2 k+2) l} N_{A_{k+1}}^{q}\left(X^{\prime}, \mathscr{F}\right)=0 .
$$

Since multiplication by $f$ maps $C^{q-1}(\mathfrak{B}, \mathscr{F})$ injectively and continuously onto a closed subspace of $C^{q-1}(\mathfrak{B}, \mathscr{F}), f \bar{B}^{q-1}(\mathfrak{B}, \mathscr{F})$ is a closed subspace of $Z^{q-1}(\mathfrak{B}, \mathscr{F})$, where $\bar{B}^{q-1}(\mathfrak{B}, \mathscr{F})$ is the topological closure of $B^{q-1}(\mathfrak{B}, \mathscr{F})$ in $C^{q-1}(\mathfrak{B}, \mathscr{F})$. By (11), $f \bar{B}^{q-1}(\mathfrak{B}, \mathscr{F}) \subset \operatorname{Im} \theta$.

Take arbitrarily $g \in \bar{B}^{q-1}(\mathfrak{B}, \mathscr{F})$. There exists a sequence $\left\{g_{\mu}\right\}$ in $B^{q-1}(\mathfrak{u}, \mathscr{F})$ whose limit is $g$. $f g_{\mu} \rightarrow f g$. By applying the open mapping theorem for Fréchet spaces to the map

$$
\theta^{-1}\left(f \bar{B}^{q-1}(\mathfrak{B}, \mathscr{F})\right) \rightarrow f \bar{B}^{q-1}(\mathfrak{B}, \mathscr{F})
$$

induced by $\theta$, we obtain $s_{\mu} \oplus t_{\mu}, s \oplus t \in Z^{q-1}(\mathfrak{u}, \mathscr{F}) \oplus C^{q-2}(\mathfrak{B}, \mathscr{F})$ such that $s_{\mu} \oplus t_{\mu} \rightarrow s \oplus t, \theta\left(s_{\mu} \oplus t_{\mu}\right)=f g_{\mu}$, and $\theta(s \oplus t)=f g$.

Take arbitrarily $h_{1} \in \Gamma\left(X^{\prime}, \mathscr{I}\right)^{l}$ and $h_{2} \in \Gamma\left(X^{\prime}, \mathscr{I}\right)^{(2 k+1) l}$. By $(8), h_{1} s_{\mu} \in B^{q-1}(\mathfrak{U}, \mathscr{F})$. Since $h_{1} s_{\mu} \rightarrow h_{1} s$, by $(10)_{k}$ we have $h_{1} h_{2} s \in B^{q-1}(\mathfrak{u}, \mathscr{F})$. Hence $h_{1} h_{2} f g \in B^{q-1}(\mathfrak{B}, \mathscr{F})$. $(10)_{k+1}$ is proved. The proposition follows from (10)r. Q.E.D.

Proposition 3. Suppose $A$ is a subvariety of a Stein complex space $X, q$ is a nonnegative integer, and $\mathscr{F}$ is a coherent analytic sheaf on $X$ such that $\operatorname{dim} A \cap$ $\bar{S}_{k+q}(\mathscr{F} \mid X-A)<k$ for $k \geqq 0$. Then $H_{A}^{q}(X, \mathscr{F})$ is a Fréchet space.

Proof. $H_{A}^{q}(X, \mathscr{F})$ being a Fréchet space is equivalent to $N_{A}^{q}(X, \mathscr{F})=0$. By Theorems 1 and $2, H_{A}^{q}(X, \mathscr{F}) \approx \Gamma\left(X, \mathscr{H}_{A}^{q} \mathscr{F}\right)$. Hence, we need only prove that the proposition is true when $X$ is replaced by some arbitrary relatively compact Stein open subset. We can therefore assume the following: 
(i) $X$ is a relatively compact open subset of a Stein complex space $\tilde{X}$,

(ii) $A=\tilde{A} \cap X$ for some subvariety $\tilde{A}$ in $\tilde{X}$,

(iii) $\mathscr{H}_{\tilde{A}}^{v}(\tilde{F})$ is coherent on $\tilde{X}$ for $0 \leqq \nu<q$.

Let $R=\Gamma(X, \mathcal{O})$. Let $\mathscr{I}$ be the ideal-sheaf on $\tilde{X}$ for $\tilde{A}$ and $I=\Gamma(\tilde{X}, \mathscr{I})$. We are going to prove (12) by induction on $q$ :

$H_{A}^{q}(X, \mathscr{F})$ is a Fréchet space and, if $\varphi: R^{p} \rightarrow H_{A}^{q}(X, \mathscr{F})$ is an $R$ homomorphism and $I^{l} \operatorname{Im} \varphi=0$ for some $l$, then $\operatorname{Im} \varphi$ is closed.

When $q=0,(12)$ is clear, because $H_{A}^{0}(X, \mathscr{F})$ is topologically isomorphic to $\Gamma\left(X, \mathscr{H}_{A}^{0} \mathscr{F}\right)$ and $\mathscr{H}_{A}^{0} \mathscr{F}$ is coherent.

Suppose $q>0$. We can assume w.l.o.g. that $\mathscr{H}_{\tilde{A}}^{\circ}(\mathscr{F})=0$.

By Theorems 1 and 2,

$$
I^{l_{1}} H_{A}^{q-1}(X, \mathscr{F})=0 \text { for some } l_{1}
$$

and there exists an $R$-epimorphism $\gamma: R^{p} \rightarrow H_{A}^{q-1}(X, \mathscr{F})$. By Proposition 2,

$$
I^{l_{2}} N_{A}^{q}(X, \mathscr{F})=0 \text { for some } l_{2} \text {. }
$$

Since $\mathscr{H}_{\tilde{A}}^{0} \mathscr{F}=0$, we can choose $f \in I^{l_{2}}$ such that the sequence

$$
0 \longrightarrow \mathscr{F} \stackrel{\lambda}{\longrightarrow} \mathscr{F} \longrightarrow \mathscr{F} \mid f \mathscr{F} \longrightarrow 0
$$

is exact on $\tilde{X}$, where $\lambda$ is defined by multiplication by $f$. We have the following exact sequence

$$
H_{A}^{q-1}(X, \mathscr{F}) \stackrel{\alpha}{\longrightarrow} H_{A}^{q-1}(X, \mathscr{F} \mid f \mathscr{F}) \stackrel{\beta}{\longrightarrow} H_{A}^{q}(X, \mathscr{F}) \stackrel{\lambda^{*}}{\longrightarrow} H_{A}^{q}(X, \mathscr{F}) .
$$

By (13) and the induction hypothesis, $H_{A}^{q-1}(X, \mathscr{F} \mid f \mathscr{F})$ is a Fréchet space and $\operatorname{Im} \alpha=\operatorname{Im} \alpha \gamma$ is closed. By (14), $N_{A}^{q}(X, \mathscr{F}) \subset \operatorname{Ker} \lambda^{*}=\operatorname{Im} \beta$. By Lemma 1(c), the map $\beta^{-1}\left(N_{A}^{q}(X, \mathscr{F})\right) \rightarrow N_{A}^{q}(X, \mathscr{F})$ induced by $\beta$ is continuous and open. Hence $N_{A}^{q}(X, \mathscr{F})$ is topologically isomorphic to the Fréchet space $\beta^{-1}\left(N_{A}^{q}(X, \mathscr{F})\right) / \operatorname{Im} \alpha$. It follows that $N_{A}^{q}(X, \mathscr{F})=0$ and $H_{A}^{q}(X, \mathscr{F})$ is a Fréchet space.

Suppose $\varphi: R^{r} \rightarrow H_{A}^{q}(X, \mathscr{F})$ is an $R$-homomorphism such that $I^{m} \operatorname{Im} \varphi=0$ for some $m$. Since $\mathscr{H}_{\mathscr{A}}^{0} \mathscr{F}=0$, we can choose $g \in I^{m}$ such that the sequence

$$
0 \longrightarrow \mathscr{F} \stackrel{\mu}{\longrightarrow} \mathscr{F} \longrightarrow \mathscr{F} / g \mathscr{F} \longrightarrow 0
$$

is exact on $\tilde{X}$, where $\mu$ is defined by multiplication by $g$. We have the following exact sequence

$$
H_{A}^{q-1}(X, \mathscr{F}) \stackrel{\sigma}{\longrightarrow} H_{A}^{q-1}(X, \mathscr{F} / g \mathscr{F}) \stackrel{\tau}{\longrightarrow} H_{A}^{q}(X, \mathscr{F}) \stackrel{\mu^{*}}{\longrightarrow} H_{A}^{q}(X, \mathscr{F}) .
$$

Since $\mu^{*} \varphi=0$, there exists an $R$-homomorphism $\psi: R^{r} \rightarrow H_{A}^{q-1}(X, \mathscr{F} / g \mathscr{F})$ such that $\tau \psi=\varphi$. Define $\nu: R^{p} \oplus R^{r} \rightarrow H_{A}^{q-1}(X, \mathscr{F} / g \mathscr{F})$ by $\nu(a \oplus b)=\sigma(\gamma(a))+\psi(b)$. By (13), $I^{m+l_{1}} \operatorname{Im} \nu=0$. By induction hypothesis, $\operatorname{Im} \nu$ is closed. Since $\operatorname{Im} \varphi$ is topologically isomorphic to $\operatorname{Im} \nu / \operatorname{Ker} \tau$, we conclude that $\operatorname{Im} \varphi$ is closed. Q.E.D. 
Proof of Main Theorem. (i) and (iii) follow from Theorems 1 and 2 and Proposition 3.

To prove (ii), we need only show that the canonical isomorphism $\lambda: \Gamma\left(X, \mathscr{H}_{A}^{\nu} \mathscr{F}\right)$ $\rightarrow H_{A}^{\nu}(X, \mathscr{F})$ has a closed graph for $0 \leqq \nu \leqq q$. To prove the closedness of the graph of $\lambda$, it suffices to show that for every relatively compact Stein open subset $X^{\prime}$ of $X$ the canonical isomorphism $\lambda^{\prime}: \Gamma\left(X^{\prime}, \mathscr{H}_{A}^{v} \mathscr{F}\right) \rightarrow H_{A \cap x^{\prime}}^{\nu}\left(X^{\prime}, \mathscr{F}\right)$ is continuous. Let $\mathcal{O}$ be the structure sheaf of $X$. There exists a sheaf-epimorphism $\mu: \mathscr{O}^{p} \rightarrow \mathscr{H}_{A}^{\nu} \mathscr{F}$ on $X^{\prime}$. The map $\tilde{\mu}: \Gamma\left(X^{\prime}, \mathcal{O}^{p}\right) \rightarrow \Gamma\left(X^{\prime}, \mathscr{H}_{A}^{\nu} \mathscr{F}\right)$ induced by $\mu$ is open. It is clear that $\lambda^{\prime} \tilde{\mu}: \Gamma\left(X^{\prime}, \mathcal{O}^{p}\right) \rightarrow H_{A \cap X^{\prime}}^{\nu}\left(X^{\prime}, \mathscr{F}\right)$ is continuous. Hence $\lambda^{\prime}$ is continuous. Q.E.D.

Remarks. (a) The arguments used in the proofs of Propositions 1, 2, and 3 can be trivially modified to give a proof of (iii) $\Rightarrow$ (ii) in Theorem 1 which is simpler than the proofs in [6] and [9].

(b) Part (ii) of the Main Theorem can also be proved directly and very easily by induction on $q$ and by considering the following commutative diagram:

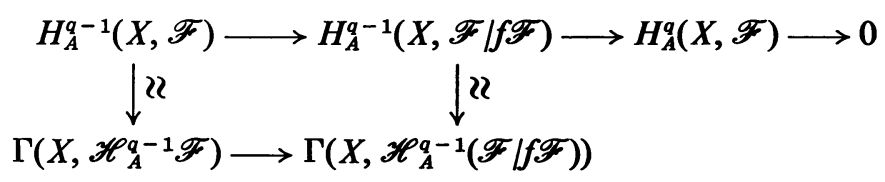

whose first row comes from the exact sequence

$$
0 \longrightarrow \mathscr{F} \stackrel{\alpha}{\longrightarrow} \mathscr{F} \longrightarrow \mathscr{F} \mid f \mathscr{F} \longrightarrow 0
$$

where $f \in \Gamma(X, \mathcal{O})$ satisfies $f_{\mathscr{H}_{A}^{q}}^{q} \mathscr{F}=0$ and $\alpha$ is defined by multiplication by $f$.

\section{REFERENCES}

1. H. Grauert, Ein Theorem der analytischen Garbentheorie und die Modulräume komplexer Strukturen, Inst. Hautes Études Sci. Publ. Math. No. 5 (1960). MR 22 \#12544.

2. R. C. Gunning and H. Rossi, Analytic functions of several complex variables, PrenticeHall, Englewood Cliffs, N. J., 1965. MR 31 \#4927.

3. G. Scheja, Riemannsche Hebbarkeitssätze für Cohomologieklassen, Math. Ann. 144 (1961), 345-360. MR 26 \#6437.

4. - Fortsetzungssätze der komplex-analytischen Cohomolgie und ihre algebraische Charakterisierung, Math. Ann. 157 (1964), 75-94. MR 31 \#738.

5. Y.-T. Siu, Analytic sheaves of local cohomology, Bull. Amer. Math. Soc. 75 (1969), 10111012.

6. - Analytic sheaves of local cohomology, Trans. Amer. Math. Soc. 148 (1970), 347-366.

7. G. Trautmann, Abgeschlossenheit von Corandmoduln und Fortsetzbarkeit kohärenter analytischer Garben, Invent. Math. 5 (1968), 216-230. MR 37 \#4289.

8. - Cohérence des faisceaux analytiques de la cohomologie locale, C.R. Acad. Sci. Paris 267 (1968), 694-695.

9. —-, Ein Endlichkeitssatz in der analytischen Geometrie, Invent. Math. 8 (1969), 143-174.

UNIVERSITY OF NOTRE DAME,

NOTRE DAME, INDIANA 46556

MATHEMATISChes Seminar deR UNiversität, Frankfurt, Federal RePUblic OF Germany 